\title{
miR-31 attenuates murine allergic rhinitis by suppressing interleukin-13-induced nasal epithelial inflammatory responses
}

\author{
FANGWEI ZHOU ${ }^{1}$, PEIQIANG LIU ${ }^{1}, \mathrm{HAO} \mathrm{LV}^{1}, \mathrm{ZIANG} \mathrm{GAO}^{1}$, WENCHUAN CHANG ${ }^{1}$ and $\mathrm{YU} \mathrm{XU}^{1,2}$ \\ ${ }^{1}$ Department of Otolaryngology-Head and Neck Surgery, Renmin Hospital of Wuhan University; \\ ${ }^{2}$ Research Institute of Otolaryngology-Head and Neck Surgery, Renmin Hospital \\ of Wuhan University, Wuhan, Hubei 430060, P.R. China
}

Received March 11, 2020; Accepted September 25, 2020

DOI: $10.3892 / \mathrm{mmr} .2020 .11680$

\begin{abstract}
The present study aimed to investigate whether microRNA (miR)-31 exerted therapeutic potential in allergic rhinitis (AR) and to explore its underlying mechanism. Firstly, the expression levels of miR-31 were detected by reverse transcription-quantitative PCR in the nasal mucosa of patients and mice. Subsequently, an ovalbumin (OVA)-induced animal model of AR was constructed. Allergic symptom score, histopathological characteristics, OVA-specific immunoglobulin E (IgE) titers, and T-helper (Th)1 and Th2 cell-related cytokine levels were analyzed in OVA-sensitized mice, miR-31-overexpressing mice, miR-negative control mice and control mice. Furthermore, interleukin (IL)-13-stimulated nasal epithelial cells (NECs) were used to assess the effects of miR-31 on the production of IL-13-induced inflammatory cytokines and mucin $5 \mathrm{AC}$ by performing western blotting and ELISA. The expression levels of miR-31 were significantly decreased in the nasal mucosa of the AR group compared with those in the control group. Moreover, upregulation of miR-31 markedly attenuated sneezing and nasal rubbing events, reduced nasal eosinophil infiltration and goblet cell hyperplasia, and decreased the levels of OVA-specific IgE and Th2-related cytokines. In addition, subsequent in vitro experiments showed that upregulation of miR-31 inhibited IL-13 receptor $\alpha 1$ chain expression and signal transducer and activator of transcription 6 phosphorylation in NECs. Furthermore, miR-31 suppressed IL-13-induced expression of thymic stromal lymphopoietin, granulocyte-macrophage colony-stimulating factor, eotaxin and mucin 5AC in NECs. In conclusion, these data revealed that miR-31 could ameliorate AR by suppressing IL-13-induced nasal epithelial inflammatory responses, and thus may serve as a novel therapeutic target for AR.
\end{abstract}

Correspondence to: Professor $\mathrm{Yu} \mathrm{Xu}$, Department of Otolaryngology-Head and Neck Surgery, Renmin Hospital of Wuhan University, 238 Jiefang Road, Wuhan, Hubei 430060, P.R. China E-mail: xuy@whu.edu.cn

Key words: allergic rhinitis, microRNA-31, interleukin-13, epithelial cell, inflammation

\section{Introduction}

Allergic rhinitis (AR) is an allergic inflammatory reaction of the nasal mucosa, and is a major health concern due to its serious impact on quality of life and work efficiency (1). AR is a global public health disease, affecting $10-40 \%$ of the population, particularly children (2). The emergence of AR symptoms is closely associated with the infiltration and activation of eosinophils, basophils, mast cells and CD4 ${ }^{+}$ $\mathrm{T}$ helper (Th) 2 cells, as well as the local release of various inflammatory mediators, which result in hyperresponsiveness, hypersecretion and remodeling of the nasal mucosa (3).

MicroRNAs (miRNAs/miRs) are a class of evolutionarily conserved endogenous non-coding RNAs, 18-25 nucleotides in length, which can negatively regulate gene expression at the post-transcriptional level by binding to target mRNA, in order to block mRNA translation or induce mRNA cleavage (4). Furthermore, miRNAs are key mediators of a variety of biological processes, including cell cycle progression, proliferation, apoptosis, angiogenesis, tumor progression and inflammation $(4,5)$. Previous studies have reported that miR-31 may have a vital role in tumor immune response, autoimmune diseases and asthma (6,7). Notably, van der Heide et al (8) demonstrated that miR-31 not only promoted the transformation of $\mathrm{CD}^{+}$ $\mathrm{T}$ cells to a Th1 phenotype, but also mediated the transcription of Th1 cytokines, prevented excessive transcription of Th2 cytokines, and maintained the Th1/Th2 balance. Recently, miR-31 has been reported to be involved in the occurrence and development of ulcerative colitis via regulating the expression of thymic stromal lymphopoietin (TSLP), a representative Th2-polarizing cytokine (9). Therefore, it was hypothesized that miR-31 might have therapeutic effects in the treatment of AR.

Previous studies have reported that upregulation of miR-31 can reduce the expression of interleukin (IL)-13 receptor $\alpha 1$ chain (IL-13R $\alpha 1$ ) and block IL-13-dependent phosphorylation of signal transducer and activator of transcription 6 (STAT6) in gut epithelium cell lines $(10,11)$. As a typical Th2 cytokine, IL-13 was shown to be a key mediator of immunoglobulin E ( $\mathrm{IgE}$ )-mediated allergic diseases via activating IL-13R $\alpha 1$ and promoting STAT6 phosphorylation (12). Furthermore, Ramalingam et al (13) reported that IL-13R $\alpha 1$-deficient mice failed to develop allergen-induced airway hyperresponsiveness and mucus hypersecretion. 
Based on the aforementioned findings, it was hypothesized that miR-31 could regulate AR by suppressing IL-13-induced nasal epithelial inflammatory responses. To the best of our knowledge, no previous study has reported the relationship between AR and miR-31, and whether miR-31 regulates AR progression remains unclear. The present study established a mouse model of AR. Subsequently, AR mice were treated with a miR-31 agomir and a series of in vitro experiments were performed to verify the hypothesis. The present findings suggested that miR-31 could serve as a novel therapeutic target for the treatment of AR. To the best of our knowledge, the present study was the first to identify the role of miR-31 in AR and to reveal the effects of miR-31 on human nasal epithelial cells (NECs). Notably, miR-31 may have similar effects on chronic inflammatory diseases of the airway, such as chronic rhinosinusitis, asthma and chronic obstructive pulmonary disease.

\section{Materials and methods}

Collection of human nasal epithelial samples. Nasal epithelial samples were collected from 10 patients with AR (age range, 18-60 years; mean age, 33.9 years) and 10 healthy subjects (age range, 20-58 years; mean age, 35.1 years) for reverse transcription-quantitative PCR (RT-qPCR). Patients were recruited at the Renmin Hospital of Wuhan University (Wuhan, China) between May 2019 and July 2019. All research subjects provided written informed consent. AR was mainly triggered by common nasal allergy triggers, such as dust mites, pollen and pet dander. AR diagnosis and treatment were carried out by physicians. Briefly, AR was diagnosed based on: i) Whether the patients had AR symptoms for $>3$ years; ii) nasal endoscopic examinations demonstrating pale and edematous nasal mucosa and watery nasal discharge; and iii) whether the patients had a positive response to the allergen test after serum antigen-specific IgE measurements (14). The exclusion criteria included acute or chronic nasal infection, and other severe systemic diseases. Patients were experiencing an allergic episode at the time of recruitment and had come to the hospital for medical assistance. Notably, no patient included in the present study had been treated with specific immunotherapy, or received antibiotics, steroids, antihistamines or immune drugs in the 4 weeks prior to recruitment $(15,16)$. The epithelial samples were gently scraped from the surface of the inferior nasal turbinate using a plastic curette and were quickly stored at $-80^{\circ} \mathrm{C}$. The study protocol complied with the Declaration of Helsinki and was approved by the Ethical Committee of Renmin Hospital of Wuhan University (approval no. WDRY2018-K020).

Animals. A total of 40 clean specific pathogen-free grade female C57BL/6 mice (weight, 20-25 g; age, 6-8 weeks) were purchased from Beijing Weitong Lihua Experimental Animal Technology Co., Ltd. [license no. SCXK (J) 2016-0006]. The mice were housed in the Animal Experiment Center of the Renmin Hospital of Wuhan University [license no. SYXK (E) 2015-0027] at room temperature (18-22 $\left.{ }^{\circ} \mathrm{C}\right)$ with a 12/12 h light/dark cycle and moderate humidity (50-60\%). The mice were reared in microisolator cages, and received free access to food and water. All mice were acclimated for a minimum of 1 week before experimentation. All protocols for animal care and handling were approved by the Institutional Animal Care and Use Committee of Renmin Hospital of Wuhan University (license no. WDRM-20190607).

AR model and intranasal administration of miR-31. The AR murine model was established as previously described (17) with minor modifications (the mice were intranasally challenged on days 21-28). The miR-31 agomir was purchased from Guangzhou RiboBio Co., Ltd. Briefly, female mice were randomly divided into four experimental groups: Control, AR, miR-31 agomir (miR-31) and miR negative control (miR-NC) groups ( $\mathrm{n}=10$ mice/group) $(18,19)$. In the AR group, mice were sensitized by intraperitoneal injection of $100 \mu \mathrm{g}$ ovalbumin (OVA, grade V; Sigma-Aldrich; Merck KGaA) and $5 \mathrm{mg}$ $\mathrm{Al}(\mathrm{OH})_{3}$ (Sigma-Aldrich; Merck KGaA) in $100 \mu$ l normal saline on days 0,7 and 14 , and were then challenged daily with OVA solution (100 mg/ml in normal saline; $20 \mu \mathrm{l} / \mathrm{mouse}$ ) into the nostrils on days 21-28. In the control group, mice were injected with the same dose of normal saline plus $\mathrm{Al}(\mathrm{OH})_{3}$ without OVA, and were then challenged with normal saline on days 21-28. In the miR-31 and miR-NC groups, mice were sensitized by intraperitoneal injection of $100 \mu \mathrm{g}$ OVA and $5 \mathrm{mg} \mathrm{Al}(\mathrm{OH})_{3}$ in $100 \mu \mathrm{l}$ normal saline on days 0,7 and 14, followed by daily intranasal administration of miR-NC or miR-31 agomir ( $25 \mu \mathrm{g} /$ nostril) $30 \mathrm{~min}$ before OVA challenge on days 21-28. A total of $24 \mathrm{~h}$ after the last challenge, all mice were sacrificed. The nasal mucosal tissues were collected for histological analysis, immunohistochemistry and RT-qPCR. Blood samples were collected from the angular vein of the mice at the time of death. The serum was harvested from blood samples by centrifugation at $4^{\circ} \mathrm{C}$ for $20 \mathrm{~min}$ at $600 \mathrm{x} \mathrm{g}$, and was stored at $-80^{\circ} \mathrm{C}$ for ELISA.

Evaluation of allergic symptoms. Within $10 \mathrm{~min}$ after the last OVA challenge, the frequency of nasal rubbing episodes and sneezing was counted for each mouse to quantitatively evaluate the symptoms of AR. If mice scratched their nose once it was defined as a single rub. To assure accurate counting of sneezes and incidences of rubbing, each mouse was observed for $10 \mathrm{~min}$ and its symptoms were recorded by three observers blinded to the experimental groups. The mean of the observations was used as the final result.

Histological analysis. The nasal mucosal tissues were removed from mice and fixed in $4 \%$ paraformaldehyde for $24 \mathrm{~h}$ at room temperature. Tissue samples were then dehydrated, embedded in paraffin and cut into $4-5 \mu \mathrm{m}$ sections. The sections were stained with hematoxylin and eosin (H\&E) and periodic acid-Schiff (PAS) for 14 days at $26^{\circ} \mathrm{C}$. The total numbers of eosinophils and goblet cells were counted in four randomly selected fields under a light microscope at x400 magnification.

Immunohistochemistry. Paraffin-embedded sections were dewaxed and endogenous peroxidase activity was blocked with $3 \% \mathrm{H}_{2} \mathrm{O}_{2}$ for $15 \mathrm{~min}$ at room temperature. Sections were then incubated with rabbit polyclonal antibodies against IL-13R $\alpha 1$ (1:1,000; cat. no. AF9095; Affinity Biosciences), STAT6 (1:1,000; cat. no. 5397; Cell Signaling Technology, Inc.) and phosphorylated (p)-STAT6 (1:1,000; cat. no. 56554; Cell 
Signaling Technology, Inc.) overnight at $4^{\circ} \mathrm{C}$, washed, incubated with goat anti-rabbit secondary antibody (1:2,000; cat. no. SA00001-2; ProteinTech Group, Inc.) for $30 \mathrm{~min}$ at room temperature, and washed again. Subsequently, samples were treated with 3,3'-diaminobenzidine solution for $5 \mathrm{~min}$ at room temperature and counterstained with hematoxylin for $30 \mathrm{sec}$ at room temperature. Positive immunoreactivity was detected by the presence of brown staining. The treated specimens were observed and analyzed under a light microscope (Olympus Corporation). Semi-quantitative analysis was performed based on the staining intensity as follows: i) 0 , colorless; ii) 1 , pale yellow; iii) 2 , brownish yellow; and iv) 3 , dark brown.

Cell culture and treatment. Human NECs were purchased from Beina Biological Company (cat. no. BNCC340481) and cultured in Dulbecco's modified Eagle's medium supplemented with $10 \%$ fetal bovine serum (both Gibco; Thermo Fisher Scientific, Inc.) and $1 \%$ penicillin/streptomycin at $37^{\circ} \mathrm{C}$ in a humidified incubator containing $5 \% \mathrm{CO}_{2}$. NECs were divided into four experimental groups: NECs, NECs + IL-13, NECs-miR-31 + IL-13 and NECs-miR-NC + IL-13 groups. The cells were cultured in medium at 70-80\% density. miR-31 mimic and miR-NC were purchased from Guangzhou RiboBio Co., Ltd. miR-31 mimic (50 nmol/1; sense, 5'-AGGCAAGAUGCUGGC AUAGCUG-3' and antisense, 3'-UCCGUUCUACCGUAUC GAC-5') and miR-NC oligonucleotides (50 nmol/1; sense, 5'-UUUGUACUACACAAAAGUACUG-3' and antisense, 3'-AAACAUGAUGUGUUUUCAUGAC-5') were transfected into NECs using riboFECT ${ }^{\mathrm{TM}} \mathrm{CP}$ Transfection kit (Guangzhou RiboBio Co., Ltd.) for $48 \mathrm{~h}$ at $37^{\circ} \mathrm{C}$. After transfection, NECs were stimulated with IL-13 (50 ng/ml; cat. no. 200-13; PeproTech, Inc.) for $24 \mathrm{~h}$ at $37^{\circ} \mathrm{C}$. The supernatants were then collected for ELISA, and cell pellets for western blot analysis.

$R T-q P C R$. Total RNA (including miRNAs) was extracted from the isolated human/mouse nasal mucosal samples and human NECs using TRIzol ${ }^{\circledR}$ reagent (Invitrogen; Thermo Fisher Scientific, Inc.), according to the manufacturer's protocol. A total of $1 \mu \mathrm{g}$ RNA was reverse transcribed into cDNA using SuperScript III Reverse Transcriptase (Invitrogen; Thermo Fisher Scientific, Inc.) at $37^{\circ} \mathrm{C}$ for $15 \mathrm{~min}$ and at $85^{\circ} \mathrm{C}$ for $5 \mathrm{sec}$. The synthesized cDNA was then amplified using Hieff ${ }^{\circledR}$ qPCR SYBR-Green Master Mix (No Rox; Shanghai Yeasen Biotechnology Co., Ltd.) on a CFX96 Touch Real-Time Detection system (Bio-Rad Laboratories, Inc.). The thermocycling conditions were as follows: $95^{\circ} \mathrm{C}$ for $10 \mathrm{~min}$, followed by 40 cycles at $95^{\circ} \mathrm{C}$ for $30 \mathrm{sec}$ and $60^{\circ} \mathrm{C}$ for $1 \mathrm{~min}$. U6 was used as the endogenous reference for miR-31. All primers were synthesized by Invitrogen; Thermo Fisher Scientific, Inc. For human samples, the primers used were as follows ( $5^{\prime}$ to $\left.3^{\prime}\right)$ : miR-31, forward CTAGGCAAGATGCTGGCATAG, reverse, CTC AACTGGTGTCGTGGAGTC; and U6, forward, CTCGCTTC GGCAGCACAT and reverse, AACGCTTCACGAATTTG CGT. For mouse samples, the primers used were as follows (5' to 3'): miR-31, forward, CTCGGATCCTGTGCATAACTG CCTTCA, reverse, CACAAGCTTGAAGTCAGGGCGAG ACAGAC; and U6, forward, CTCGCTTCGGCAGCACA and reverse, AACGCTTCACGAATTTGCGT. Each assay was run in triplicate and relative gene expression was calculated using the $2^{-\Delta \Delta \mathrm{Cq}}$ method (18).
ELISA. The concentrations of total IgE, interferon (IFN)- $\gamma$ (cat. no. DIF50C), IL-12 (cat. no. SM1270), IL-4 (cat. no. M4000B), IL-5 (cat. no. M5000) and IL-13 (cat. no. M1300CB) in mouse serum were determined using mouse ELISA kits (all purchased from R\&D Systems, Inc.). The levels of granulocyte-macrophage colony-stimulating factor (GM-CSF; cat. no. DGM00; R\&D Systems, Inc.), eotaxin (cat.no. DTX00; R\&D Systems, Inc.) and mucin 5AC [MUC5AC; cat. no. ELK2098; ELK (Wuhan) Biotechnology Co., Ltd.] in the culture supernatants were measured using human ELISA kits. ELISA kits were used according to the manufacturer's instructions.

Western blotting. NECs were homogenized and lysed in RIPA lysis buffer (Sigma-Aldrich; Merck KGaA) with protease and phosphatase inhibitors. Protein concentration was determined using BCA reagent (Beyotime Institute of Biotechnology). Protein samples $(40 \mu \mathrm{g})$ were separated by SDS-PAGE on $12 \%$ gel, and subsequently transferred onto polyvinylidene difluoride membranes. The membranes were then blocked in $5 \%$ low-fat milk for $1 \mathrm{~h}$ at room temperature, following which the membranes were incubated with primary antibodies at $4^{\circ} \mathrm{C}$ overnight, followed by incubation with a goat anti-rabbit secondary antibody (1:10,000; cat. no. 926-32211; LI-COR Biosciences) at room temperature for $2 \mathrm{~h}$. The membranes were washed with TBS with $0.1 \%$ Tween-20 and scanned using an Odyssey infrared scanning device (LI-COR Biosciences). The Odyssey ${ }^{\circledR}$ CLx Imaging System (LI-COR Biosciences) was used to detect the expression level of each protein. Rabbit anti-mouse TSLP $(1: 1,000)$, STAT6 $(1: 1,000)$, p-STAT6 $(1: 1,000)$ and GAPDH $(1: 1,000)$ antibodies were purchased from Cell Signaling Technology, Inc. Rabbit anti-mouse IL-13R $\alpha 1(1: 1,000)$ antibodies was purchased from Affinity Biosciences. Band intensities (gray values) were measured with ImageJ 1.52 (National Institutes of Health), and GAPDH was used as the internal reference.

Statistical analysis. All data analyses were performed with SPSS 23.0 software (IBM Corp.) and GraphPad Prism 6.0 software (GraphPad Software, Inc.). Descriptive analysis, normality test and homogeneity test of variance were performed for all data sets. The Kolmogorov-Smirnov test and Bartlett test were used to test normality and homogeneity of variance, respectively. Data with normal distribution were presented as mean \pm standard deviation. Multiple groups were compared using one-way analysis of variance followed by Tukey's post hoc test, and two groups were compared using the Student's t-test. Data in Fig. 4 are presented as the median (IQR), and differences among groups were compared using Kruskal-Wallis followed by a Dunn's post hoc test. $\mathrm{P}<0.05$ was considered to indicate a statistically significant difference.

\section{Results}

miR-31 is significantly downregulated in nasal mucosa from patients with $A R$ and AR mice. As shown in Fig. 1A, the expression levels of miR-31 in nasal mucosa were significantly downregulated in patients with AR compared with those in healthy individuals. In addition, the expression levels of miR-31 were also significantly downregulated in the in the nasal mucosa of AR mice compared with those in control 
A Human

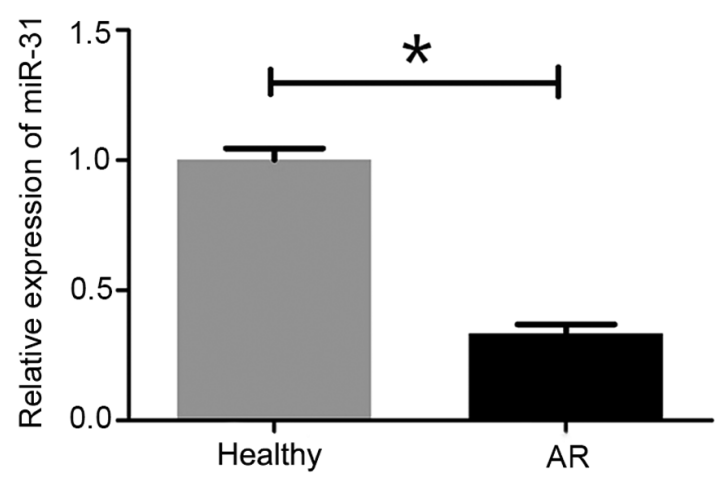

B Mouse

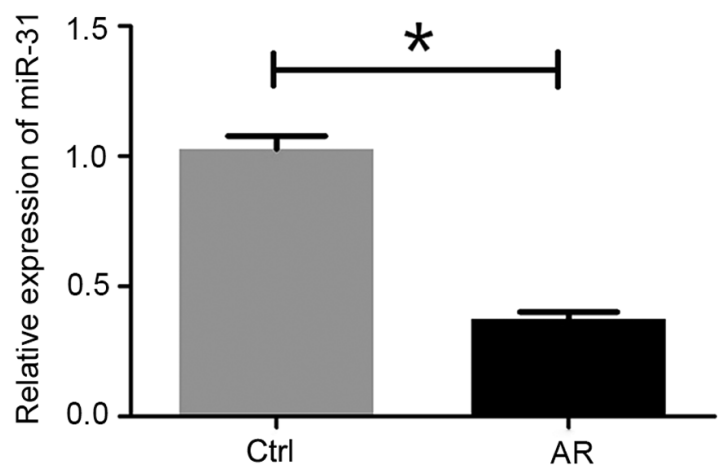

Figure 1. miR-31 expression levels were decreased in the nasal mucosa of patients and mice with AR. Nasal epithelial samples were collected from (A) patients with AR $(n=10)$ and healthy subjects $(n=10)$, and $(B)$ control $(n=10)$ and AR $(n=10)$ groups. The expression levels of miR-31 were analyzed by reverse transcription-quantitative polymerase chain reaction. Data are presented as the mean $\pm \mathrm{SD}$. ${ }^{*} \mathrm{P}<0.05$. AR, allergic rhinitis; miR-31, microRNA-31; NC, negative control.
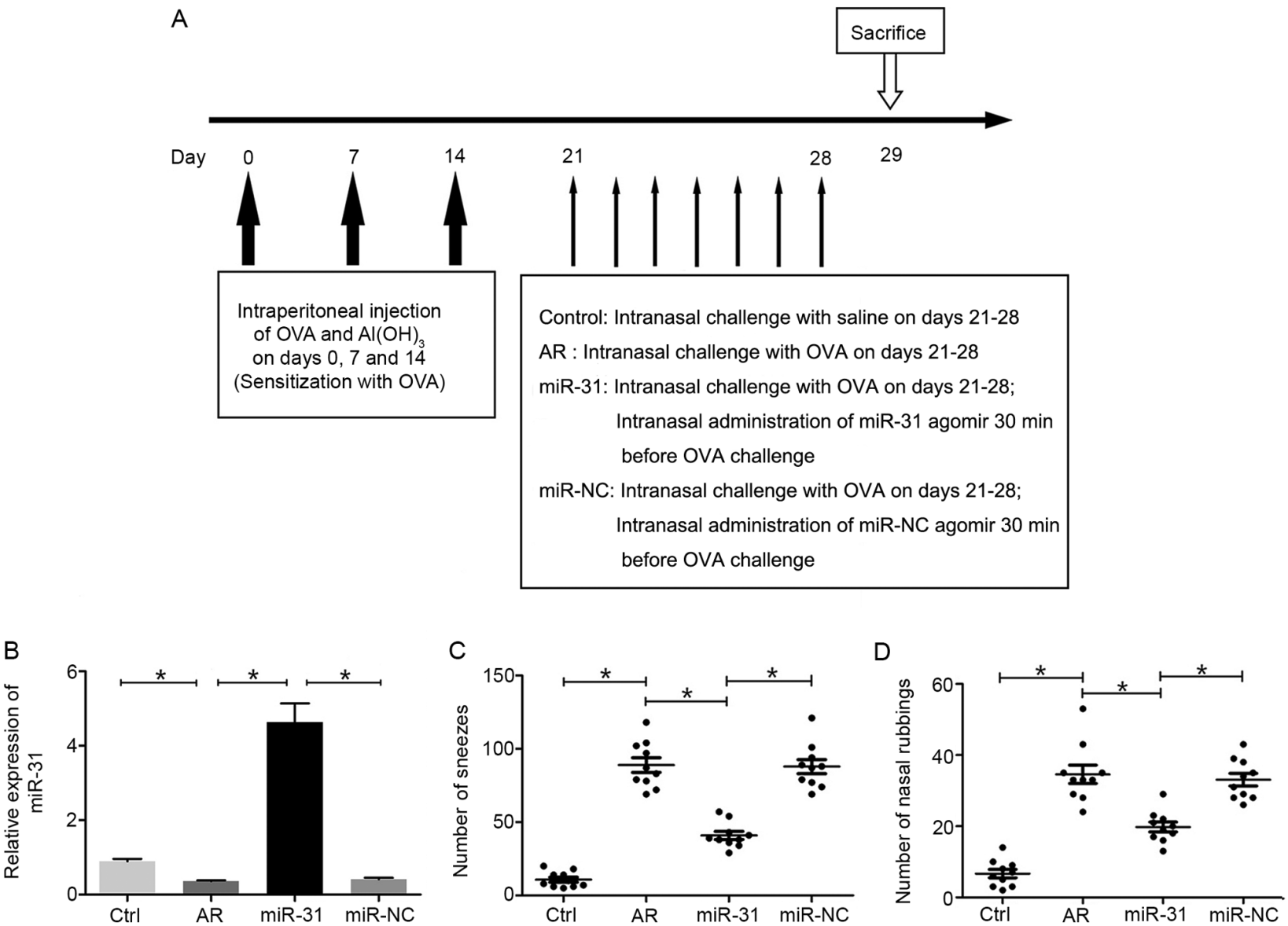

Figure 2. Intranasal administration of miR-31 attenuates OVA-induced allergic symptoms. (A) OVA and miR-31 agomir administration protocol. (B) Expression levels of miR-31 in mice nasal mucosal tissues was determined by reverse transcription-quantitative polymerase chain reaction. Number of (C) sneezes and (D) nasal rubbing events were counted in the indicated groups $(\mathrm{n}=10)$. Data are presented as the mean $\pm \mathrm{SD}$. * $\mathrm{P}<0.05$. AR, allergic rhinitis; OVA, ovalbumin; miR-31, microRNA-31; NC, negative control; Ctrl, control.

mice, thus confirming the finding that miR-31 expression was markedly downregulated under AR conditions (Fig. 1B).

miR-31 alleviates allergic symptoms, and reduces nasal eosinophil infiltration and goblet cell hyperplasia in mice. The present study assessed the effect of intranasal administration of miR-31 on AR symptoms in mice (Fig. 2A). To confirm whether exogenous miR-31 entered mouse nasal mucosal tissues, the expression levels of miR-31 were detected in nasal mucosal tissues. The results revealed that intranasal administration with miR-31 agomir significantly upregulated the expression levels of miR-31 in nasal mucosal tissues. Intranasal administration with miR-NC had no influence on the expression of miR-31 (Fig. 2B). As shown in Fig. 2C and $\mathrm{D}$, the number of sneezes and nose rubbing events were significantly increased in OVA-induced mice compared with those in 
A

Ctr

AR

miR-31

miR-NC

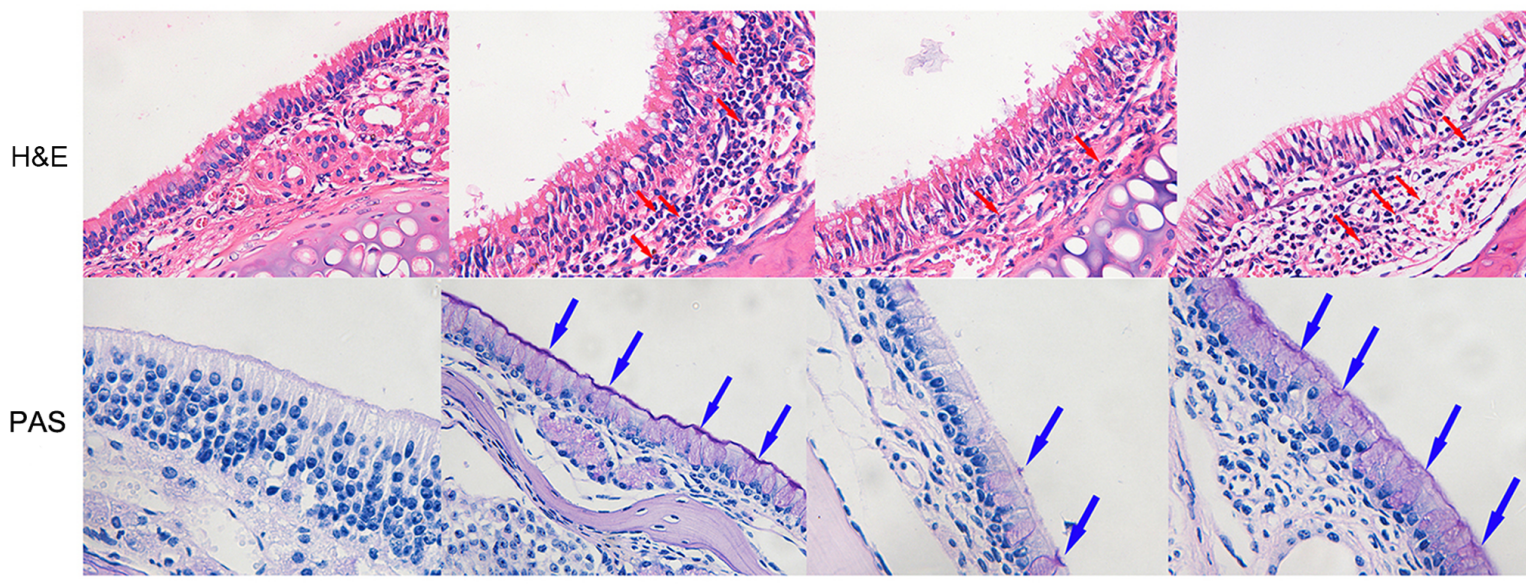

B
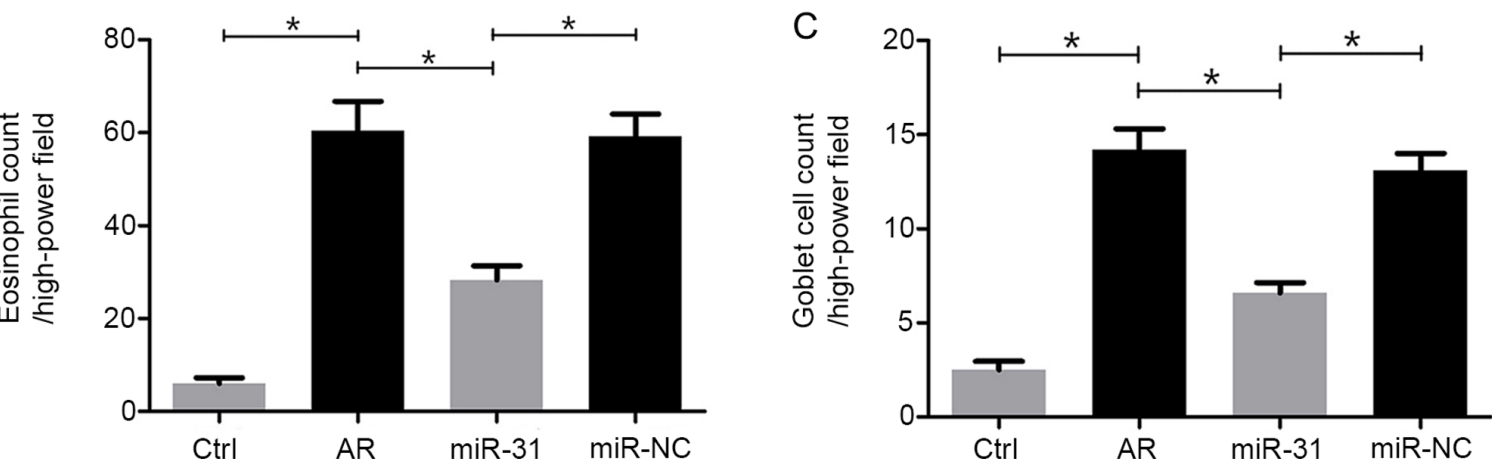

Figure 3. Histopathological changes in the nasal mucosa of mice as detected by H\&E and PAS staining. (A) The cytoplasm of eosinophils in the nasal lamina propria were stained red by H\&E. Red arrows indicate eosinophils. The goblet cells in the nasal epithelial surface were stained red by PAS stain. Blue arrows indicate PAS-positive goblet cells. Statistical analysis for (B) eosinophils and (C) goblet cells in the nasal mucosa. The numbers of eosinophils and goblet cells were counted under a microscope at $\mathrm{x} 400$ magnification. All data are presented as the mean $\pm \mathrm{SD}$ ( $\mathrm{n}=6 / \mathrm{group}$ ). ${ }^{*} \mathrm{P}<0.05$. AR, allergic rhinitis; miR-31, microRNA-31; NC, negative control; Ctrl, control; H\&E, hematoxylin and eosin; PAS, periodic acid-Schiff.

control mice. In addition, in the AR and miR-NC groups, both sneezing and nasal rubbings were observed more frequently than those in the miR-31 group. Therefore, these data demonstrated that intranasal administration of miR-31 significantly alleviated allergic symptoms in AR mice.

The present study also analyzed the pathological changes in the nasal mucosal tissues of mice by histological analysis. OVA-induced mice exhibited histopathological features of AR, including cilia shedding, numerous eosinophils, goblet cell hyperplasia and monocyte recruitment in the nasal mucosa. The number of eosinophils and goblet cells were markedly higher in OVA-induced mice compared with those in control mice. By contrast, miR-31 agomir-treated mice displayed fewer eosinophils and goblet cells than mice in the AR and miR-NC groups. No significant differences in AR histopathological features were observed between the AR and miR-NC groups (Fig. 3). These findings suggested that miR-31 agomir exhibited anti-inflammatory activity to reduce eosinophil infiltration and goblet cell hyperplasia in the nasal mucosa.

miR-31 inhibited IL-13Ral expression and STAT6 phosphorylation in vivo. The present study evaluated the protein expression levels of IL-13R $\alpha 1$, p-STAT6 and STAT6 in nasal mucosal tissues by immunohistochemical analysis. The results revealed that overexpression of miR-31 inhibited the expression of IL-13R $\alpha 1$ and phosphorylation of STAT6 in vivo (Fig. 4).
miR-31 decreases serum levels of total IgE in AR mice. To investigate the effect of miR-31 on total IgE levels, the levels of serum total IgE were measured by ELISA. As shown in Fig. 5A, total IgE levels were markedly higher in OVA-induced mice compared with those in control mice. Following miR-31 treatment, OVA-induced mice exhibited significantly reduced levels of total IgE. These data demonstrated that miR-31 may regulate the levels of total $\mathrm{IgE}$ in AR mice.

miR-31 decreases serum cytokine concentrations in $A R$ mice. To assess the effect of miR-31 on AR-associated inflammation, the serum levels of several inflammatory cytokines were determined by ELISA. As shown in Fig. 5B-D, the levels of the Th2 cytokines, IL-4, IL-5 and IL-13, were significantly increased in AR mice compared with those in control mice, and administration of exogenous miR-31 significantly decreased their levels. Conversely, the reduced levels of the Th1 cytokines IFN- $\gamma$ and IL-12 in AR mice were partially corrected following miR-31 agomir treatment (Fig. 5E and F). These results suggested that miR-31 may exert an anti-AR role via regulating the secretion of inflammatory cytokines.

miR-31 inhibits IL-13Ral expression and STAT6 phosphorylation in human NECs. Gain-of-function experiments were performed in human NECs using miR-31 mimics. To 

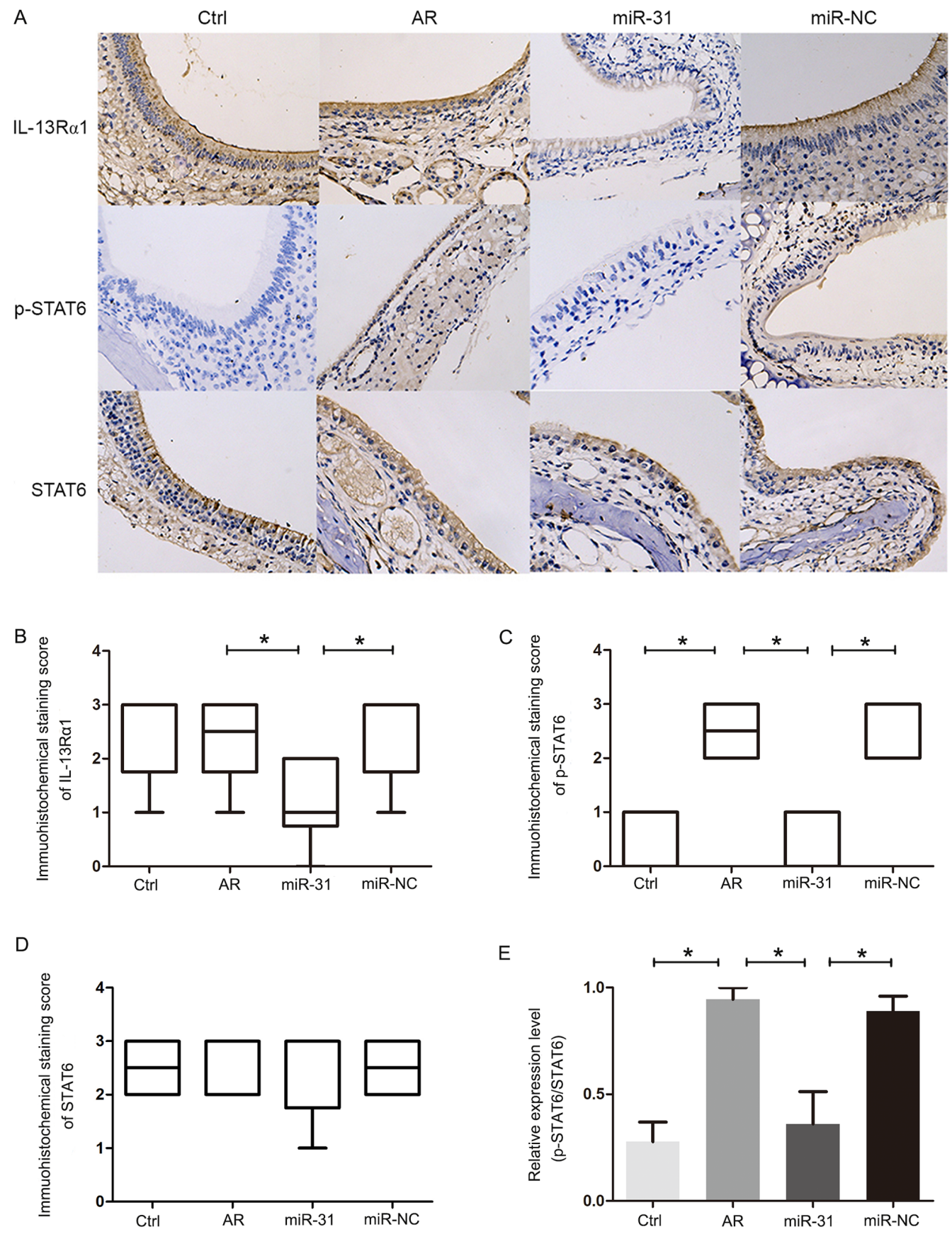

Figure 4. Expression of IL-13R $\alpha 1$, p-STAT6 and STAT6 in nasal mucosal tissues. IL-13R $\alpha 1$, p-STAT6 and STAT6 expression was detected by immunohistochemical staining. Nasal mucosal tissues from different groups were subjected to single staining using IL-13R $\alpha 1$, p-STAT6 and STAT6 antibodies. (A) Immunohistochemical staining image (original magnification, $x 400$ ). (B-D) Immunohistochemical staining scores. (E) Relative expression level of p-STAT6/STAT6. All data are presented as the median (IQR) (n=6/group). Differences among groups were compared using Kruskal-Wallis followed by a Dunn's post hoc test. "P<0.05. AR, allergic rhinitis; miR-31, microRNA-31; NC, negative control; Ctrl, control; IL-13R $\alpha 1$, interleukin-13 receptor $\alpha 1$ chain; STAT6, signal transducer and activator of transcription 6; p-STAT6, phosphorylated-STAT6.

confirm whether exogenous miR-31 entered human NECs, the mRNA expression levels of miR-31 were detected in each experimental group. The results revealed that transfection with miR-31 mimics significantly upregulated the expression of miR-31 in NECs (Fig. 6A). To confirm whether miR-31 can inhibit IL-13R $\alpha 1$ expression and STAT6 phosphorylation in human NECs, western blotting was performed on protein samples prepared from NECs in each treatment group. The results demonstrated that overexpression of miR-31 significantly suppressed IL-13R $\alpha 1$ expression (Fig. 6B) and had an inhibitory effect on STAT6 phosphorylation (Fig. 6C).
miR-31 suppresses IL-13-induced expression of TSLP, GM-CSF, eotaxin and MUC5AC in human NECs. To confirm whether miR-31 regulates the synthesis of inflammatory cytokines and MUC5AC protein in IL-13-stimulated NECs, TSLP protein expression levels were measured in the cell pellets, and GM-CSF, eotaxin and MUC5AC levels were measured in the culture supernatants of each group. The results demonstrated that the protein expression levels of TSLP, and the levels of GM-CSF, eotaxin and MUC5AC were significantly increased in NECs following IL-13 stimulation for $24 \mathrm{~h}$, but were significantly decreased in miR-31-overexpressing NECs 
A

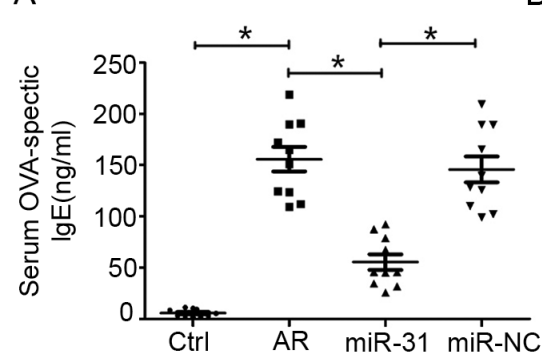

$B$

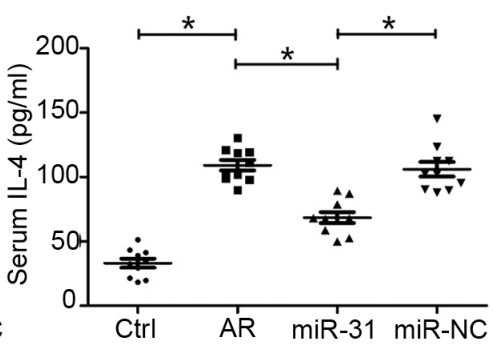

$\mathrm{E}$
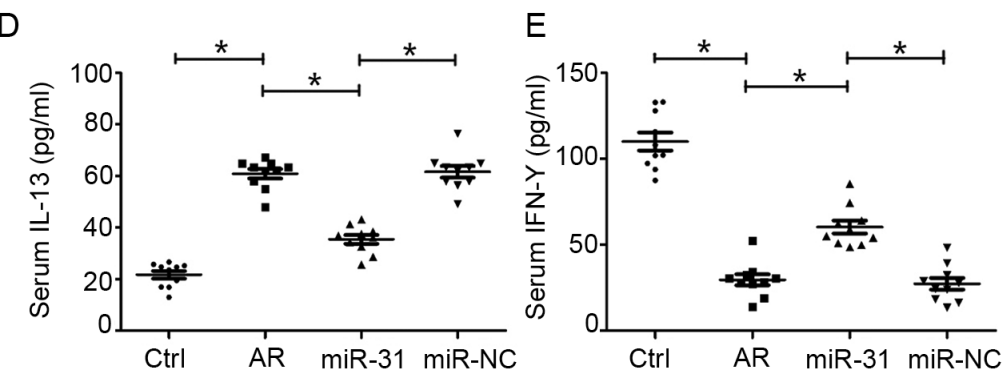

C
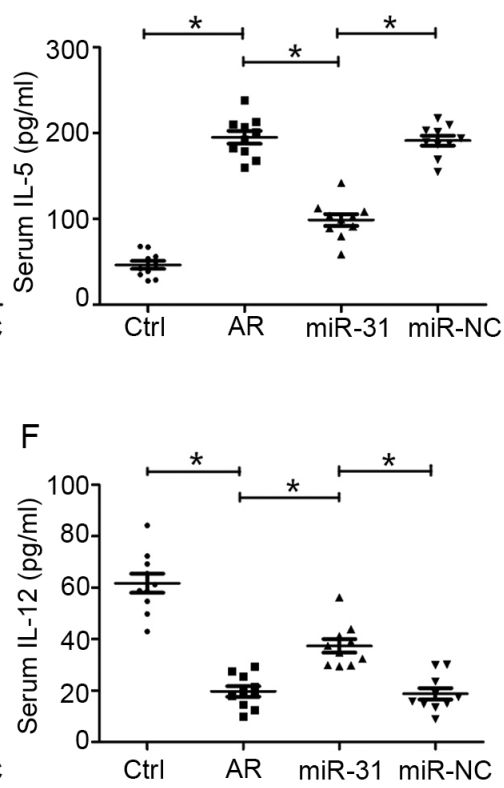

Figure 5. Effects of miR-31 on the production of OVA-specific IgE and inflammatory cytokines. Overexpression of miR-31 affected the secretion of (A) OVA-specific IgE, and the inflammatory cytokines, (B) IL-4, (C) IL-5, (D) IL-13, (E) IFN- $\gamma$ and (F) IL-12, in the serum of AR mice. ELISA was performed to detect the protein concentrations of these cytokines. Data are presented as the mean \pm SD of 10 mice in each group. ${ }^{*} \mathrm{P}<0.05$. AR, allergic rhinitis; miR-31, microRNA-31; NC, negative control; Ctrl, control; IL, interleukin; IgE, immunoglobulin E; IFN- $\gamma$, interferon- $\gamma$; OVA, ovalbumin.

compared with those in the cells treated with IL-13 only. In addition, no obvious differences were observed between the IL-13-stimulated and miR-NC groups (Fig. 7).

\section{Discussion}

AR is a chronic inflammatory disease of the nasal mucosa involving a variety of immune cells, cytokines and inflammatory mediators. Routine AR treatments include antihistamines, antileukotrienes agents and glucocorticoid-based drugs, which only provide short-term relief from AR symptoms $(19,20)$. In addition, some patients do not respond to these pharmacotherapies. Therefore, it is important to improve understanding of the pathogenesis of AR and to develop improved treatment strategies. Increasing evidence has suggested that AR development is a genetic manipulation process, and miRNAs have been shown to act as key regulators in allergic diseases (21). As suppressors of gene expression at the post-transcriptional level, miRNAs have been reported to serve a vital role in the development and activity of innate and adaptive immune systems, which are involved in inflammation, and hence are considered promising diagnostic and prognostic biomarkers and treatment targets (22).

A previous study reported that miR-31 may have an important role in various inflammation-related disorders, such as psoriasis, systemic lupus erythematosus, inflammatory bowel disease and autoimmune encephalomyelitis (23). miR-31 was previously revealed to be overexpressed in the keratinocytes of patients with psoriasis, and was shown to increase the expression of cytokines and chemokines, leading to chronic inflammation (24). Moreover, miR-31 has been reported to regulate the function of $\mathrm{T}$ cells in patients with systemic lupus erythematosus (7). Whiteoak et al (9) demonstrated that TSLP served a role in promoting mucosal healing and regulating inflammation in ulcerative colitis, whereas miR-31 could inhibit this effect. However, little is known about the role of miR-31 in AR.

The present study demonstrated that the expression levels of miR-31 were significantly lower in the nasal mucosa of patients with AR and AR mice compared with those in the control groups, thus suggesting that miR-31 negatively regulated pro-allergic properties in AR. The results of in vivo experiments revealed that treatment of mice with a miR-31 agomir significantly alleviated allergic symptoms, such as sneezing and nasal rubbing, suggesting its potential in AR treatment. It has been reported that IgE-mediated immune responses, inflammatory cell disorders and Th1/Th2 imbalance-induced abnormal cytokine production are closely related to the occurrence of AR, and there is a critical link between them with regards to AR pathogenesis (25). The present results also demonstrated that overexpression of miR-31 effectively ameliorated AR pathology through inhibition of local inflammatory responses, including eosinophil infiltration and goblet cell hyperplasia. In addition, consistent with previous findings, serum OVA-specific IgE concentration was decreased, and the secretion of anti-inflammatory cytokines (IFN- $\gamma$ and IL-12) mainly produced by Th1 cells (correcting the Th1/Th2 imbalance) were increased following intranasal administration of miR-31 (19). As the nose has an abundant supply of blood and good lymphatic circulation (26), nasal administration was used in the present study for quick absorption into systemic circulation. The systemic immune change may be caused by blood circulation or lymphatic circulation after the intranasal administration of the miRNA; however, the mechanism needs to be further studied. Taken together, these findings suggested that miR-31 may have a therapeutic effect on an experimental AR model.

Previous studies have demonstrated that NECs are not only effector cells in AR, but also have an important role 
A

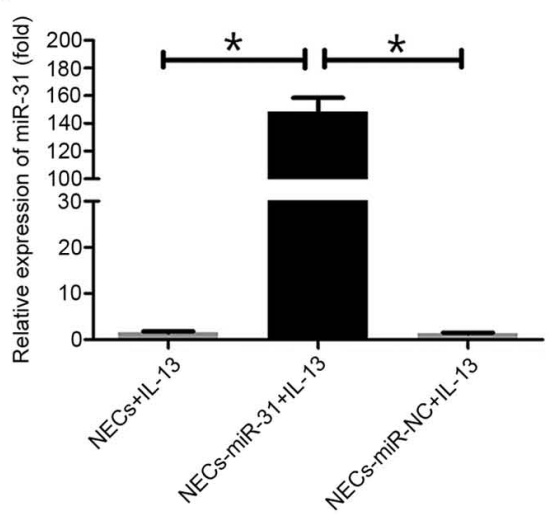

B
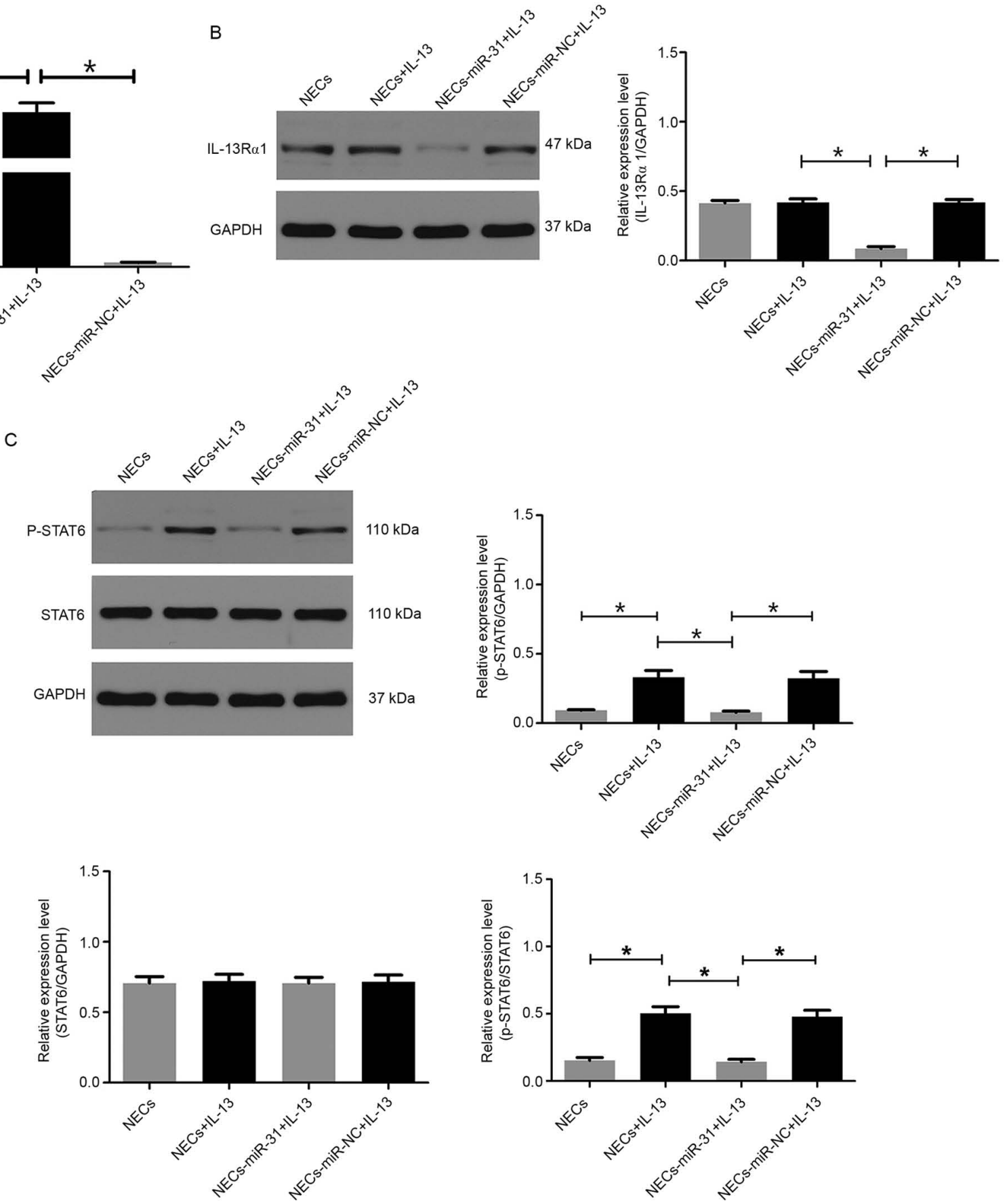

Figure 6. miR-31 inhibits IL-13R 1 expression and STAT6 phosphorylation in human NECs. (A) Expression level of miR-31 in different groups was determined by reverse transcription-quantitative polymerase chain reaction $(\mathrm{n}=6)$. (B) miR-31 overexpression suppressed the protein expression levels of IL-13R 1 , as determined by western blotting $(\mathrm{n}=3)$. (C) Transfection with miR-31 mimics had an inhibitory effect on STAT6 phosphorylation, as indicated by western blotting ( $\mathrm{n}=3$ ). GAPDH was used as the internal control. All data are presented as the mean \pm SD. "P<0.05. NECs, nasal epithelial cells; miR-31, microRNA-31; NC, negative control; IL-13, interleukin-13; IL-13R $\alpha 1$, IL-13 receptor $\alpha 1$ chain; STAT6, signal transducer and activator of transcription 6; p-STAT6, phosphorylated-STAT6.

in the development of airway allergic inflammation, and are involved in the production of GM-CSF and polarization of allergen-driven Th2 lymphocytes $(27,28)$. In previous studies, NECs have been shown to be a rich source of proinflammatory cytokines, such as tumor necrosis factor- $\alpha$, eotaxin, GM-CSF, IL-6 and IL-8, which serve a central role in airway host defense, asthma and AR $(29,30)$. IL-13 is a typical Th2-related cytokine that promotes airway hyperresponsiveness, inflammation, mucus production and fibrosis, and participates in allergic reactions in each stage of attack (16).
Recent studies have shown that IL-13 and IL-13-induced signaling pathways in AR are closely associated with increased mucus production and secretion of inflammatory mediators in airway epithelial cells $(16,31)$. Furthermore, it has been demonstrated that IL-13 may induce pathological responses in airway allergic diseases, such as eosinophil recruitment, mucus cell metaplasia, subepithelial fibrosis and smooth muscle hypertrophy $(30,32)$. Hence, based on these findings, IL-13-treated NECs were used to induce an AR-like phenotype in vitro. 

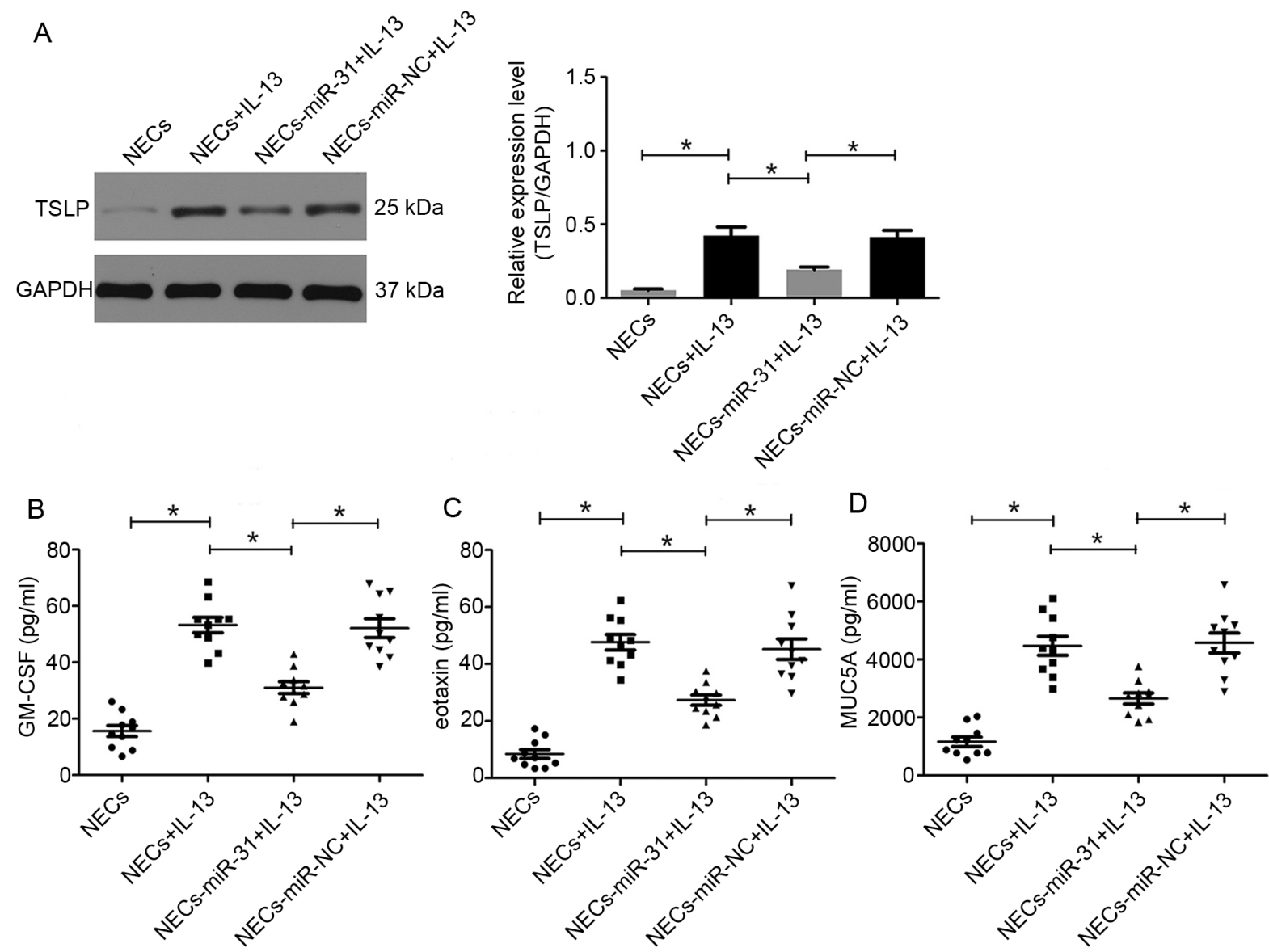

Figure 7. Effects of miR-31 overexpression on the production of inflammatory cytokines and MUC5AC protein in IL-13-stimulated NECs. (A) Protein expression levels of TSLP were measured by western blotting $(n=3)$. GAPDH was used as the internal control. The protein levels of (B) GM-CSF, (C) eotaxin and (D) MUC5AC were measured by ELISA ( $\mathrm{n}=10)$. miR-31 overexpression suppressed IL-13-induced expression of TSLP, GM-CSF, eotaxin and MUC5AC in human NECs. All data are presented as the mean \pm SD. "P $<0.05$. NECs, nasal epithelial cells; miR-31, microRNA-31; NC, negative control; IL-13, interleukin-13; TSLP, thymic stromal lymphopoietin; GM-CSF, granulocyte-macrophage colony-stimulating factor; MUC5A, mucin 5AC.

A previous study revealed that miR-31 has an important role in limiting IL-13 signaling in ulcerative colitis (11). Therefore, blocking the IL-13 signaling pathway using miR-31 mimics may be a beneficial approach for controlling the pathological process of allergic airway diseases. IL-13 signaling is initiated by binding with IL-13R $\alpha 1$, which forms a receptor complex with IL-4R $\alpha$ and mediates signal transduction through eliciting phosphorylation of STAT6 $(33,34)$. In addition, IL-13R $\alpha 1$ is a critical signaling molecule and is essential for the development of allergen-induced airway inflammation (13). STAT6, an important signaling molecule for IL-13-induced inflammatory response, is usually expressed in airway epithelial cells and has an important role in the development of allergic airway inflammation (35). It has also been reported that activation of STAT6 is critical for IL-13-induced TSLP, eotaxin and MUC5AC expression in epithelial cells during OVA-induced airway inflammation $(36,37)$. Therefore, the present study performed western blotting to detect the protein expression levels of IL-13R $\alpha 1$ and p-STAT6. Transfection with miR-31 mimics significantly decreased IL-13R $\alpha 1$ expression and STAT6 phosphorylation compared with in cells transfected with miR-NC, suggesting that miR-31 may inhibit IL-13 signaling in NECs. To the best of our knowledge, this is the first study reporting the effects of miR-31 on human NECs.
TSLP, an epithelium-derived cytokine, is regarded as a novel pro-allergic molecule. TSLP can strongly activate dendritic cells through interaction with the TSLP receptor to induce an inflammatory Th2-type response, which is essential for initiating allergic inflammation (38). It has been demonstrated that IL-13 can induce TSLP production in the nasal epithelium in a STAT6-dependent manner, which is critical for the development of IL-13-driven allergic inflammation in the nasal mucosa (37). As pro-inflammatory cytokines in allergic airway inflammation, GM-CSF and eotaxin are synthesized and released by airway epithelial cells, infiltrating leukocytes and fibroblasts in response to allergens and inflammatory mediators $(39,40)$. GM-CSF can stimulate the differentiation of hematopoietic stem cells into granulocytes and monocytes, and support eosinophil survival (41). Eotaxins are a family of CC chemokines which recruit immune cells, such as Th2 cells, eosinophils, mast cells and basophils to inflammatory sites in allergic diseases (31). Additionally, MUC5AC is a glycoprotein belonging to the superfamily of mucins. Mucus hypersecretion is a common feature of AR (42). The present results revealed that miR-31 overexpression significantly decreased the protein expression levels of TSLP in NECs, and the levels of GM-CSF, eotaxin and MUC5AC secreted by NECs following IL-13 stimulation. These data suggested that the anti-inflammatory effect of miR-31 on AR may be mediated by suppressing 
inflammatory responses in the nasal epithelium via inhibiting IL-13-induced inflammatory cytokine and mucus production.

However, this study has several limitations. Firstly, although scraping and collection of human nasal epithelial cells is not particularly invasive, this action often makes patients uncomfortable, thus limiting the clinical sample size of the present study. In future studies, more patients and volunteers will be recruited. Secondly, the dosage of miR-31 agomir was chosen based a previous study protocol (43). In future, the optimal dosage of miR-31 agomir should be tested to determine the most effective dosage. Thirdly, although IL-13-induced inflammatory response in the nasal epithelium is a crucial part of AR pathology, other pathways may be involved in miR-31-mediated effects in AR, which requires further investigation.

In conclusion, the present study demonstrated the suppressive effects of intranasal administration of miR-31 on allergic airway responses in an OVA-induced mouse AR model. The results demonstrated that miR-31 could alleviate allergic symptoms and suppress IL-13 signaling in nasal mucosal. Furthermore, these data indicated that miR-31 might exert its anti-inflammatory effects on AR through inhibiting IL-13-induced inflammatory cytokine and mucus production in NECs. Hence, miR-31 may serve as a potential therapeutic target for AR.

\section{Acknowledgements}

Not applicable.

\section{Funding}

This study was supported by the National Natural Science Foundation of China (grant nos. 81371070 and 81770986) and the Wuhan Science and Technology Bureau Scientific Research Project (grant no. 2016060101010037)

\section{Availability of data and materials}

The datasets used and/or analyzed during the current study are available from the corresponding author on reasonable request.

\section{Authors' contributions}

FZ conceived and designed the current study, performed the experiments and wrote the manuscript. PL and HL analyzed the data. WC and ZG contributed to study design and manuscript preparation. YX conceived the study and critically reviewed the manuscript. All authors read and approved the final manuscript.

\section{Ethics approval and consent to participate}

Written informed consent was obtained from all the patients and the research protocols were approved by the Ethics Committee of Renmin Hospital of Wuhan University.

\section{Patient consent for publication}

Not applicable.

\section{Competing interests}

The authors declare that they have no competing interests.

\section{References}

1. Brożek JL, Bousquet J, Agache I, Agarwal A, Bachert C, Bosnic-Anticevich S, Brignardello-Petersen R, Canonica GW, Casale T, Chavannes $\mathrm{NH}$, et al: Allergic rhinitis and its impact on asthma (ARIA) guidelines-2016 revision. J Allergy Clin Immunol 140: 950-958, 2017.

2. Katelaris $\mathrm{CH}$, Lee BW, Potter PC, Maspero JF, Cingi C, Lopatin A, Saffer M, Xu G and Walters RD: Prevalence and diversity of allergic rhinitis in regions of the world beyond Europe and North America. Clin Exp Allergy 42: 186-207, 2012.

3. Scadding G: Cytokine profiles in allergic rhinitis. Curr Allergy Asthma Rep 14: 435, 2014.

4. Almeida MI, Reis RM and Calin GA: MicroRNA history: Discovery, recent applications, and next frontiers. Mutat Res 717: 1-8, 2011.

5. Bartel DP: MicroRNAs: Target recognition and regulatory functions. Cell 136: 215-233, 2009.

6. Li L, Hui Y and Xing C: MicroRNA-31 affects the expression of asthma-related cytokines via regulation of CD44. Int J Clin Exp Med 9: 11, 2016.

7. Fan W, Liang D, Tang Y, Qu B, Cui H, Luo X, Huang X, Chen S, Higgs BW, Jallal B, et al: Identification of microRNA-31 as a novel regulator contributing to impaired interleukin-2 production in $\mathrm{T}$ cells from patients with systemic lupus erythematosus. Arthritis Rheum 64: 3715-3725, 2012.

8. van der Heide V, Möhnle P, Rink J, Briegel J and Kreth S: Down-regulation of MicroRNA-31 in $\mathrm{CD}^{+} \mathrm{T}$ cells contributes to immunosuppression in human sepsis by promoting $\mathrm{TH} 2$ skewing. Anesthesiology 124: 908-922, 2016.

9. Whiteoak SR, Claridge A, Balendran CA, Harris RJ, Gwiggner M, Bondanese VP, Erlandsson F, Hansen MB, Cummings JR and Sanchez-Elsner T: MicroRNA-31 targets thymic stromal lymphopoietin in mucosal infiltrated $\mathrm{CD} 4^{+} \mathrm{T}$ cells: A Role in Achieving Mucosal Healing in Ulcerative Colitis? Inflamm Bowel Dis 24: 2377-2385, 2018

10. Gwiggner M, Claridge A, Cummings F and Sanchez-Elsner T: OC-162 the role of micrornas miR-31 and miR-155 in the deregulation of the IL-13 pathway in ulcerative colitis. Gut 61 (Suppl 2): A69-A70, 2012.

11. Gwiggner M, Martinez-Nunez RT, Whiteoak SR, Bondanese VP, Claridge A, Collins JE, Cummings JR and Sanchez-Elsner T: MicroRNA-31 and MicroRNA-155 Are Overexpressed in ulcerative colitis and regulate IL-13 signaling by targeting interleukin 13 receptor $\alpha-1$. Genes (Basel) 9: 85, 2018.

12. Niu Y, Murata T, Watanabe K, Kawakami K, Yoshimura A, Inoue J, Puri RK and Kobayashi N: MIP-T3 associates with IL-13Ralpha1 and suppresses STAT6 activation in response to IL-13 stimulation. FEBS Lett 550: 139-143, 2003.

13. Ramalingam TR, Pesce JT, Sheikh F, Cheever AW, Mentink-Kane MM, Wilson MS, Stevens S, Valenzuela DM, Murphy AJ, Yancopoulos GD, et al: Unique functions of the type II interleukin 4 receptor identified in mice lacking the interleukin 13 receptor alphal chain. Nat Immunol 9: 25-33, 2008.

14. Cheng L, Chen J, Fu Q, He S, Li H, Liu Z, Tan G, Tao Z, Wang D, Wen $\mathrm{W}$, et al: Chinese society of allergy guidelines for diagnosis and treatment of allergic rhinitis. Allergy Asthma Immunol Res 10: 300-353, 2018.

15. Tan L, Qiu T, Xiang R, Cao C, Deng Y, Tao Z and Xu Y: Down-regulation of Tet2 is associated with Foxp3 TSDR hypermethylation in regulatory $\mathrm{T}$ cell of allergic rhinitis. Life Sci 241: 117101,2020

16. Wang L, Lv Q, Song X, Jiang K and Zhang J: ADRB2 suppresses IL-13-induced allergic rhinitis inflammatory cytokine regulated by miR-15a-5p. Hum Cell 32: 306-315, 2019.

17. Xiang R, Xu Y, Zhang W, Kong YG, Tan L, Chen SM, Deng YQ and Tao ZZ: Semaphorin 3A inhibits allergic inflammation by regulating immune responses in a mouse model of allergic rhinitis. Int Forum Allergy Rhinol 9: 528-537, 2019.

18. Livak KJ and Schmittgen TD: Analysis of relative gene expression data using real-time quantitative PCR and the 2(- $\Delta \Delta \mathrm{C}(\mathrm{T}))$ method. Methods 25: 402-408, 2001.

19. Wang J, Cui Z, Liu L, Zhang S, Zhang Y, Zhang Y, Su H and Zhao Y: MiR-146a mimic attenuates murine allergic rhinitis by downregulating TLR4/TRAF6/NF- $\kappa$ B pathway. Immunotherapy 11: 1095-1105, 2019. 
20. Liu HJ, Zhang AF, Zhao N and Li XZ: Role of miR-146a in enforcing effect of specific immunotherapy on allergic rhinitis. Immunol Invest 45: 1-10, 2016.

21. Singh PB, Pua HH, Happ HC, Schneider C, von Moltke J, Locksley RM, Baumjohann D and Ansel KM: MicroRNA regulation of type 2 innate lymphoid cell homeostasis and function in allergic inflammation. J Exp Med 214: 3627-3643, 2017.

22. Lu TX and Rothenberg ME: Diagnostic, functional, and therapeutic roles of microRNA in allergic diseases. J Allergy Clin Immunol 132: 3-13, quiz 14, 2013.

23. Stepicheva NA and Song JL: Function and regulation of microRNA-31 in development and disease. Mol Reprod Dev 83: 654-674, 2016.

24. $\mathrm{Xu} \mathrm{N}$, Meisgen F, Butler LM, Han G, Wang XJ, Söderberg-Nauclér C, Ståhle M, Pivarcsi A and Sonkoly E: MicroRNA-31 is overexpressed in psoriasis and modulates inflammatory cytokine and chemokine production in keratinocytes via targeting serine/threonine kinase 40. J Immunol 190 678-688, 2013

25. Yu S, Han B, Liu S, Wang H, Zhuang W, Huang Y and Zhang R: Derp1-modified dendritic cells attenuate allergic inflammation by regulating the development of T helper type1(Th1)/Th2 cells and regulatory $\mathrm{T}$ cells in a murine model of allergic rhinitis. Mol Immunol 90: 172-181, 2017

26. Cunha S, Amaral MH, Lobo JMS and Silva AC: Lipid nanoparticles for nasal/intranasal drug delivery. Crit Rev Ther Drug Carrier Syst 34: 257-282, 2017.

27. Shimizu S, Kouzaki H, Ogawa T, Takezawa K, Tojima I and Shimizu T: Eosinophil-epithelial cell interactions stimulate the production of MUC5AC mucin and profibrotic cytokines involved in airway tissue remodeling. Am J Rhinol Allergy 28: 103-109, 2014

28. Wang Y, Bai C, Li K, Adler KB and Wang X: Role of airway epithelial cells in development of asthma and allergic rhinitis Respir Med 102: 949-955, 2008.

29. Adam E, Coulon L and Jaumotte E: The house dust mite protease allergen Der 1 induced IL- 8 production in human airway epithelial cells through activation of ERK1/2 mitogen-activated protein kinase and AP-1 signaling pathways. J Allergy Clin Immunol 113: S339-S339, 2004.

30. Matsukura S, Stellato C, Georas SN, Casolaro V, Plitt JR, Miura K, KurosawaS,SchindlerU and SchleimerRP: Interleukin-13 upregulates eotaxin expression in airway epithelial cells by a STAT6-dependent mechanism. Am J Respir Cell Mol Biol 24: 755-761, 2001

31. Shi J, Luo Q, Chen F, Chen D, Xu G and Li H: Induction of IL-6 and IL- 8 by house dust mite allergen Der p1 in cultured human nasal epithelial cells is associated with PAR/PI3K/NFkappaB signaling. ORL J Otorhinolaryngol Relat Spec 72: 256-265, 2010.

32. Bartel S, La Grutta S, Cilluffo G, Perconti G, Bongiovanni A, Giallongo A, Behrends J, Kruppa J, Hermann S, Chiang D, et al: Human airway epithelial extracellular vesicle miRNA signature is altered upon asthma development. Allergy 75: 346-356, 2020.
33. O'Shea JJ, Gadina M and Schreiber RD: Cytokine signaling in 2002: New surprises in the Jak/Stat pathway. Cell 109 (Suppl 1): S121-S131, 2002.

34. Kuperman D, Schofield B, Wills-Karp M and Grusby MJ: Signal transducer and activator of transcription factor 6 (Stat6)-deficient mice are protected from antigen-induced airway hyperresponsiveness and mucus production. J Exp Med 187: 939-948, 1998.

35. Urban JF Jr, Noben-Trauth N, Donaldson DD, Madden KB Morris SC, Collins M and Finkelman FD: IL-13, IL-4Ralpha, and Stat6 are required for the expulsion of the gastrointestinal nematode parasite Nippostrongylus brasiliensis. Immunity 8: 255-264, 1998.

36. Miyata M, Nakamura Y, Shimokawa N, Ohnuma Y, Katoh R Matsuoka S, Okumura K, Ogawa H, Masuyama K and Nakao A: Thymic stromal lymphopoietin is a critical mediator of IL-13-driven allergic inflammation. Eur J Immunol 39: 3078-3083, 2009.

37. Wang X, Li Y, Luo D, Wang X, Zhang Y, Liu Z, Zhong N, Wu M and Li G: Lyn regulates mucus secretion and MUC5AC via the STAT6 signaling pathway during allergic airway inflammation. Sci Rep 7: 42675, 2017.

38. Takai T: TSLP expression: Cellular sources, triggers, and regulatory mechanisms. Allergol Int 61: 3-17, 2012.

39. Teng Y, Zhang R, Liu C, Zhou L, Wang H, Zhuang W, Huang Y and Hong Z: miR-143 inhibits interleukin-13-induced inflammatory cytokine and mucus production in nasal epithelial cells from allergic rhinitis patients by targeting IL13R $\alpha 1$. Biochem Biophys Res Commun 457: 58-64, 2015.

40. Matsuwaki Y, Wada K, White T, Moriyama $\mathrm{H}$ and Kita $\mathrm{H}$ : Alternaria fungus induces the production of GM-CSF, interleukin- 6 and interleukin- 8 and calcium signaling in human airway epithelium through protease-activated receptor 2. Int Arch Allergy Immunol 158 (Suppl 1): 19-29, 2012.

41. Daffern PJ, Jagels MA, Saad JJ, Fischer W and Hugli TE: Upper airway epithelial cells support eosinophil survival in vitro through production of GM-CSF and prostaglandin E2: Regulation by glucocorticoids and TNF-alpha. Allergy Asthma Proc 20: 243-253, 1999.

42. Tomazic PV, Darnhofer B and Birner-Gruenberger R: Nasal mucus proteome and its involvement in allergic rhinitis. Expert Rev Proteomics 17: 191-199, 2020.

43. Zhang XH, Zhang YN, Li HB, Hu CY, Wang N, Cao PP, Liao B, Lu X, Cui YH and Liu Z: Overexpression of miR-125b, a novel regulator of innate immunity, in eosinophilic chronic rhinosinusitis with nasal polyps. Am J Respir Crit Care Med 185: $140-151,2012$

This work is licensed under a Creative Commons Attribution-NonCommercial-NoDerivatives 4.0 International (CC BY-NC-ND 4.0) License. 